\title{
Activation of the central melanocortin system in rats persistently reduces body and fat mass independently of caloric reduction
}

\begin{tabular}{|r|l|}
\hline Journal: & Canadian Journal of Physiology and Pharmacology \\
\hline Manuscript ID & cjpp-2017-0440.R1 \\
\hline Date Submitted by the Author: & 15-Aug-2017 \\
\hline Complete List of Authors: & $\begin{array}{l}\text { Côté, Isabelle; University of Florida, Pharmacology and Therapeutics } \\
\text { Green, Sara; University of Florida, Pharmacology and Therapeutics } \\
\text { Morgan, Drake; University of Florida, Psychiatry } \\
\text { Carter, Christy; University of Florida, Aging and Geriatric Research } \\
\text { Tümer, Nihal; University of Florida, Pharmacology and Therapeutics } \\
\text { Scarpace, Philip; University of Florida, Pharmacology and Therapeutics }\end{array}$ \\
\hline $\begin{array}{r}\text { Is the invited manuscript for } \\
\text { consideration in a Special } \\
\text { Issue?: }\end{array}$ & N/A \\
\hline Keyword: & Melanotan II, body mass, body fat, lean/fat mass ratio, Caloric reduction \\
\hline \multicolumn{2}{|c}{} \\
\hline
\end{tabular}


Title: Activation of the central melanocortin system in rats persistently reduces body and fat mass independently of caloric reduction

Isabelle Cote $^{1}$, Sara M. Green ${ }^{1}$, Drake Morgan ${ }^{2}$, Christy S. Carter ${ }^{3}$, Nihal Tümer ${ }^{1}$, and Philip J. Scarpace $^{1}$

${ }^{1}$ Department of Pharmacology and Therapeutics, University of Florida, Gainesville, Florida

${ }^{2}$ Department of Psychiatry, University of Florida, Gainesville, Florida

${ }^{3}$ Department of Aging and Geriatric Research, University of Florida, Gainesville, Florida

University of Florida

Department of Pharmacology and Therapeutics

1200 Newell Drive

Gainesville, FL 32610

Author to whom correspondence should be sent:

Philip J. Scarpace

scarpace@ufl.edu 


\begin{abstract}
Recent evidence indicate that Melanotan II (MTII) reduces body mass independently of caloric reduction. Because MTII induces a transient hypophagia, caloric reduction is still considered a primary mechanism for MTII-mediated body mass loss. To examine the contribution of caloric reduction to long-term body mass loss in response to Melanotan II (MTII), we centrally infused MTII or vehicle in ad libitum fed (MTII and Control) animals in comparison to a group of animals that were pair-fed (PF) to the MTII group. Food intake and body mass were recorded daily, and body composition was assessed biweekly. The present study demonstrates that central MTII-mediated body mass loss is only partially mediated by caloric restriction, and the longterm body mass loss is independent of the initial hypophagia. More importantly, central MTII administration induced a rapid but sustained fat mass loss, independently of caloric reduction. MTII-treated animals preserved their lean/fat mass ratio throughout the study whereas PF animals underwent a transient reduction of lean/fat mass ratio that was only normalized when food intake returned to Control level. In summary, it can be concluded that activation of the central melanocortin system in rats persistently reduces body and fat mass independently of caloric reduction.
\end{abstract}




\section{Introduction}

The central melanocortin system modulates energy homeostasis predominantly through the proopiomelanocortin (POMC) cleavage product, $\alpha$-melanocyte-stimulating hormone ( $\alpha-\mathrm{MSH})$, which suppresses food intake and energy storage (Ellacott and Cone 2004). The bioactive peptide $\alpha$-MSH acts on central melanocortin receptors 3 and $4\left(\mathrm{MC}_{3 / 4}\right.$ receptors $)$ to modulate the activity of a neural circuit inducing hypophagia and a strong catabolic response. Due to the rapid inactivation of $\alpha-\mathrm{MSH}$ in vivo ( 20 minutes), melanotropic molecules resistant to endogenous degradation have been developed (Wallingford et al. 2009) such as Melanotan II (MTII), a potent $\alpha$-MSH synthetic analog. MTII non-selectively activate MC receptors and rapidly induces body mass loss through, at least in part, reducing caloric intake (Li et al. 2004; Pierroz et al. 2002). Central administration of MTII induces a consistent hypophagic response decreasing food consumption by up to $50 \%$ of initial food consumption. However, appetite is normalized to baseline within the first few days of MTII (Lucas et al. 2015; Zhang et al. 2010).

We recently showed that chronic central infusion of MTII persistently reduces body mass while only transiently inducing hypophagia, indicating that the central melanocortin system may be a long-term regulator of energy balance without the necessity of maintaining caloric restriction over time (Cote et al. 2017). However, without a control group with matched caloric intake, the contribution of the initial caloric reduction to the long-term body mass loss could not be excluded. Thus, hypophagia is still considered a primary mechanism of MTII-induced catabolism (Bluher et al. 2004; Pierroz et al. 2002). Because appetite is normalized within a few days of central MTII treatment, we postulated that body mass reduction does not depend solely upon caloric restriction. To verify our hypothesis, MTII or vehicle was centrally infused over 4 
weeks into ad libitum fed (MTII and Control) rats, and compared with animals that were pair-fed with the MTII group (PF). Food intake and body mass were recorded daily, and body composition was assessed biweekly by TD-NMR. 


\section{Methods}

\section{Animals}

Ten-month old male Fisher $344 \times$ Brown Norway $(\mathrm{F} 344 \mathrm{BN})$ rats $(n=15)$ weighing from $360 \mathrm{~g}$ to 480g were obtained from the National Institute on Aging Colony at Charles River Laboratories (Wilmington, MA). Upon arrival, animals were housed individually on a 12-h light and 12-h dark cycle. All rats were allowed a week to acclimate to their new environment before beginning any surgical procedure. Rats were fed a standard rodent chow (Envigo Teklad Global diet 2918, Madison, WI). Health status, body weight, and food intake were monitored daily. All experimental protocols were approved by the University of Florida's Animal Care and Use Committee, and in compliance with the "Guide for the Care and Use of Laboratory Animals".

\section{Experimental time line}

Three groups of five rats were used. The Control and Pair-fed (PF) groups received a vehicle central infusion and one group received MTII. The PF rats were given the average amount of food consumed daily by the MTII-treated rats (lagging one day behind). A successful implantation was confirmed by the initial hypophagia phase by MTII. One rat from the MTII group remained non-responsive to the treatment and was discarded and excluded from food intake calculation starting day 10. All the data recorded from that rat was removed, hence the slight difference in daily food intake prior to that day. 


\section{Surgeries}

Rats were anaesthetized with isoflurane (2.5\%) and administered the analgesics Buprenorphine (0.025 mg kg-1; SC) and Carprofen (5 mg kg-1; SC) daily for 72 hours, starting prior the surgery. All animals were first infused with artificial cerebrospinal fluid (aCSF; $\mathrm{NaCl} 148 \mathrm{mM}$, $\mathrm{KCl} 3 \mathrm{mM}, \mathrm{CaCl} 2-2 \mathrm{H} 2 \mathrm{O} 1.5 \mathrm{mM}, \mathrm{MgCl} 2-6 \mathrm{H} 2 \mathrm{O} 1.4 \mathrm{mM}, \mathrm{Na} 2 \mathrm{HPO} 41.5 \mathrm{mM}$, NaH2PO4 $0.2 \mathrm{mM})$ through a cannula implanted into the lateral ventricle using a stereotaxic device $(1.3 \mathrm{~mm}$ posterior to bregma and $1.9 \mathrm{~mm}$ lateral to the midsagittal suture and to a depth of $3.5 \mathrm{~mm}$ ). The cannula was connected to an osmotic mini-pump (Durect Corporation, Cupertino, CA). Original mini-pumps were replaced after recovery from the surgery through a small incision $(1 \mathrm{~cm})$. Fresh aCSF or MTII ( $2 \mu \mathrm{g}$ /day diluted in aCSF; Genscript, NJ, USA) was administered for 28 days. MTII was previously reported to be stable at $37^{\circ} \mathrm{C}$ for 28 days (Jonsson et al. 2002).

\section{Determination of body composition using time-domain nuclear magnetic resonance}

Body composition was determined biweekly using time-domain nuclear magnetic resonance (TD-NMR; Minispec, Bruker Optics, The Woodlands, TX). The MiniSpec quantifies three components of body composition (fat mass, free body fluid, and lean body mass). The TD-NMR analyzes signals from all protons in the sample area. The final values comprised the average of two scans for each animal.

\section{Statistical analyses}

Results are expressed as means with their standard errors. Differences between means were tested for statistical significance $(P<0.05)$ using a one-way ANOVA with repeated measures for longitudinal analyses or non-repeated measures for single time-point analyses. For body 
composition assessments, a two-way ANOVA (mixed model) was used. In case of significant event $(P<0.05)$, Bonferroni's multiple comparison test was applied to determine individual differences between means. 


\section{Results}

Central MTII infusion-mediated body mass loss is only partially mediated by caloric restriction Consistent with our previous report (Cote et al. 2017), central infusion of MTII induced a robust and sustained body mass loss. Indeed, MTII-treated animals lost more body mass than Control rats (Fig. 1A: $P<0.001)$. In the PF group, there was an initial body mass loss, however this was not maintained and body mass returned to control rats' levels by day 28 (Fig. 1A: $P<0.001$ ). Nadir of body mass loss was recorded in the middle of the study (Day 15 and 16), and was 60\% greater in MTII than in PF animals (Fig. 1A: $-44.7 \pm 2.3 \mathrm{~g}$ vs $-28.1 \pm 2.9 \mathrm{~g}$ ). Food intake rapidly

dropped over the few first days of MTII treatment but returned to Control levels by day 9 (Fig. 1B). Despite the rapid normalization of food intake, longitudinal statistical analyses showed that overall daily food intake from day 0 to 28 was significantly reduced in MTII and PF animals (Fig. 1B: Control vs MTII/PF; $P s<0.001)$ but there was no difference in food intake between MTII and PF groups. Similarly, when food consumption was normalized as gram consumed per gram of body mass, MTII and PF animals ate significantly less than Control animals (Fig. 1C: $P s<0.01)$ but MTII and PF groups consumed the same amount of food.

\section{MTII delays body mass regain following transient caloric restriction}

Longitudinal analyses indicated a significant reduction in daily food consumption. However, additional statistical tests showed that the hypophagia occurred only within the first two weeks of treatment (Fig. 2A: Control vs MTII/PF: $P<0.01$ ). During this hypophagic phase, both MTII and PF groups lost body mass compared with Control (Fig. 2D: Control vs MTII/PF: $P<0.01$ ). There were no significant differences in food consumption during the last two weeks of 
treatment (Fig. 2B). Surprisingly, despite the significant reduction in food consumption between day 0 and 14 in MTII and PF groups, food intake consumed throughout the study was similar across groups (Fig. 2C). More importantly, although there were no significant differences in cumulative food intake (Fig. 2C) or daily food intake across groups (Fig. 1B), only the MTII group displayed significant reduction in body mass by the end of the study (Fig. 2F: Control vs MTII: $P<0.001$; Control vs PF: $P>0.05$; MTII vs PF: $P<0.05)$.

\section{Central MTII induced a rapid and robust fat mass loss independently of caloric restriction}

During the first two weeks of infusion, MTII rats lost significantly more fat mass than Control rats (Fig. 3A: $P<0.001$ ). Unexpectedly, fat mass loss was 10 -fold greater in MTII than in PF rats (Fig. 3A: $-22.2 \pm 1.4 \mathrm{~g}$ vs $-2.1 \pm 2.2 \mathrm{~g} ; P<0.001$ ). Interestingly, during the period when the PF rats were restricted to less than ad libitum food intake level (first two weeks) fat mass in PF rats did not differ from those of Control rats, suggesting that body mass loss following short-term caloric restriction does not necessarily target fat mass (Fig 3A). MTII rats regained some of their lost fat mass over the two last weeks of treatment; however, fat mass remained significantly lower in MTII than and Control rats (Fig. 3A: $P<0.01$ ). At endpoint, MTII-mediated fat mass loss persisted and was 2-fold more in MTII than in PF rats (Fig. 3A: - $19.7 \pm 1.8 \mathrm{~g}$ vs $-10.8 \pm 2.8$ $\mathrm{g} ; P<0.05)$.

\section{MTII maintained whereas caloric restriction transiently deteriorated body composition}

MTII-treated group did not only lose fat mass but lost substantial amount of lean body mass. At day 14, MTII rats had lost a significant amount of lean body mass whereas Control animals remained stable (Fig. 3B: - $16.1 \pm 1.2$ vs $-0.6 \pm 2.0 ; P<0.001$ ). During this hypophagic period, 
the corresponding PF rats also lost lean body mass compared with Control rats (Fig. 3B: $P<0.05)$. By day 28, delta lean body mass was lower in MTII (Fig. 3B: Control vs MTII; $P<0.05$ ) but not in PF animals (Fig. 3B: Control vs PF; $P>0.05$ ). Despite the significant decrease in lean body mass in MTII rats, lean/fat mass ratio increased from day 0 to day 14 to a level that was not statistically different from Control (Fig. 3C). In PF animals, lean/fat mass ratio was decreased at day 14 corresponding to the end food restriction period (Fig. 3C: Control vs PF and MTII vs PF; Ps $<0.05$ ), but was normalized to Control level by day 28 . From day 14 to 28 , PF rats consumed the same amount of food as Control rats or nearly ad libitum food levels. 


\section{Discussion}

Activation of the central melanocortin system in rats induced a more robust and sustained body mass and fat mass loss than did transient caloric reduction alone. Indeed, MTII-treated animals lost $60 \%$ more body mass than PF rats. We hereby demonstrate that long-term reduction of body mass loss by the central melanocortin system is not dependent upon the initial hypophagia. More importantly, the present study demonstrates that the activation of the central melanocortin system rapidly and persistently reduces fat mass independently of caloric reduction.

MTII-treated animals underwent a transient hypophagia that dissipated by day 14 . However, the initial reduction in food intake did not significantly affect the total amount of food consumed animals display enhanced body mass loss compared with PF animals (Choi et al. 2003; Li et al. 2004) and that MTII chronically reduces body mass without the necessity of maintaining caloric restriction (Cote et al. 2017; Hamilton and Doods 2002), the present study provides an extension of the previous knowledge by demonstrating that long-term reduction of body mass in MTIItreated animals is independent of caloric reduction. MTII has been shown to increase energy expenditure through, at least in part, enhancing oxygen consumption (Hamilton and Doods 2002).

However, the contribution of energy expenditure to the long-lasting body mass reduction has never been ascertained. Our report provides indirect evidence that increased energy expenditure significantly contributed to sustained body mass loss. In line with this interpretation, PF animals but not MTII rats essentially recovered their body mass by the end of the study. In published studies, because observation of MTII and pair-fed animals was limited to the hypophagic phase, 
this conclusion could not be drawn. Furthermore, longitudinal analyses of body composition uncovered that MTII induces a rapid yet persistent fat mass loss that is also independent of caloric reduction. Our study is the first to delineate the nature of long-term body mass loss under chronic MTII treatment that are independent of caloric reduction. The present study shows that MTII animals exhibited lower delta fat mass than Control and PF rats throughout the study, whereas no differences between PF and Control animals were observed at any time point. These data also extend previous findings in which MTII-treated rats displayed lower fat mass than Control pair-fed rats following 6-day of treatment, corresponding to the initial hypophagic phase (Li et al. 2004). The present study shows that MTII-treated animals had reduced fat mass compared with PF rats and differences in fat mass were observed beyond the hypophagic phase, thus demonstrating that MTII-mediated long-term fat loss is independent of caloric reduction. In contrast, a previous study conducted in mice reported that hypophagia is the primary mechanism for MTII-induced weight loss because MTII-treated mice did not lose more body mass than control pair-fed mice (Pierroz et al. 2002). The discrepancy between our results could be due to differences in rodent model or in experimental design. Animals from that study were fed a high fat diet, and dietary fats were shown to attenuate the anorexic effects of central MTII (Clegg et al. 2003). Finally, another novel finding of the present study is that MTII does not negatively affect body composition as observed when simply reducing caloric intake. While both MTII and PF groups lost a significant amount of lean body mass, the PF group displayed a transient reduction of lean/fat mass ratio that recovered after food intake returned to Control level.

In summary, our study demonstrates that the long-term reduction of body mass in MTII-treated animals is independent of caloric reduction. More importantly, longitudinal analyses of body 
composition enabled to determine that the rapid yet persistent fat mass loss by MTII is also independent of caloric reduction. Finally, our study also shows for the first time that, although MTII decreases lean body mass, the lean/fat mass ratio is maintained throughout the study. These data indicate that the central melanocortin system plays an important role in the long-term regulation of energy balance through mechanisms other than just caloric reduction. 


\section{References}

Bluher, S., Ziotopoulou, M., Bullen, J.W., Jr., Moschos, S.J., Ungsunan, L., Kokkotou, E., Maratos-Flier, E., and Mantzoros, C.S. 2004. Responsiveness to peripherally administered melanocortins in lean and obese mice. Diabetes 53(1): 82-90.

Choi, Y.H., Li, C., Hartzell, D.L., Lin, J., Della-Fera, M.A., and Baile, C.A. 2003. MTII administered peripherally reduces fat without invoking apoptosis in rats. Physiol Behav 79(2): $331-337$.

Clegg, D.J., Benoit, S.C., Air, E.L., Jackman, A., Tso, P., D'Alessio, D., Woods, S.C., and Seeley, R.J. 2003. Increased dietary fat attenuates the anorexic effects of intracerebroventricular injections of MTII. Endocrinology 144(7): 2941-2946. doi: 10.1210/en.2002-0218.

Cote, I., Sakarya, Y., Kirichenko, N., Morgan, D., Carter, C.S., Tumer, N., and Scarpace, P.J. 2017. Activation of the central melanocortin system chronically reduces body mass without the necessity of long-term caloric restriction. Can J Physiol Pharmacol 95(2): 206-214. doi: 10.1139/cjpp-2016-0290.

Ellacott, K.L., and Cone, R.D. 2004. The central melanocortin system and the integration of short- and long-term regulators of energy homeostasis. Recent Prog Horm Res 59: 395-408.

Hamilton, B.S., and Doods, H.N. 2002. Chronic application of MTII in a rat model of obesity results in sustained weight loss. Obes Res 10(3): 182-187. doi: 10.1038/oby.2002.28.

Jonsson, L., Skarphedinsson, J.O., Skuladottir, G.V., Watanobe, H., and Schioth, H.B. 2002. Food conversion is transiently affected during 4-week chronic administration of melanocortin agonist and antagonist in rats. J Endocrinol 173(3): 517-523. 
Li, G., Zhang, Y., Wilsey, J.T., and Scarpace, P.J. 2004. Unabated anorexic and enhanced thermogenic responses to melanotan II in diet-induced obese rats despite reduced melanocortin 3 and 4 receptor expression. J Endocrinol 182(1): 123-132.

Lucas, N., Legrand, R., Breton, J., Dechelotte, P., Edwards-Levy, F., and Fetissov, S.O. 2015. Chronic delivery of alpha-melanocyte-stimulating hormone in rat hypothalamus using albuminalginate microparticles: effects on food intake and body weight. Neuroscience 290: 445-453. doi: 10.1016/j.neuroscience.2015.01.037.

Pierroz, D.D., Ziotopoulou, M., Ungsunan, L., Moschos, S., Flier, J.S., and Mantzoros, C.S. 2002. Effects of acute and chronic administration of the melanocortin agonist MTII in mice with diet-induced obesity. Diabetes 51(5): 1337-1345.

Wallingford, N., Perroud, B., Gao, Q., Coppola, A., Gyengesi, E., Liu, Z.W., Gao, X.B., Diament, A., Haus, K.A., Shariat-Madar, Z., Mahdi, F., Wardlaw, S.L., Schmaier, A.H., Warden, C.H., and Diano, S. 2009. Prolylcarboxypeptidase regulates food intake by inactivating alphaMSH in rodents. J Clin Invest 119(8): 2291-2303. doi: 10.1172/JCI37209.

Zhang, Y., Collazo, R., Gao, Y., Li, G., and Scarpace, P.J. 2010. Intermittent MTII application evokes repeated anorexia and robust fat and weight loss. Peptides 31(4): 639-643. doi: 10.1016/j.peptides.2009.12.019. 


\section{Figure legends}

\section{Fig. 1 Longitudinal changes in body mass and daily food intake}

Control (black circles), MTII (open circles), and PF (gray circles). Food intake and body mass were recorded daily throughout the study. Body composition was assessed by TD-NMR at day 0 , 14, and 28 and marked on the graph. A Cumulative changes in body mass in grams (g). There were significant differences between all groups. Both MTII and PF resulted in significantly higher reduction in body mass $(P<0.001)$ relative to Control group. MTII treated animals lost a significantly higher amount of body mass than PF $(P<0.001)$ B Daily food consumption in grams during the course of the experiment. Both MTII and PF had reduced caloric intake relative to Control group $(P<0.001)$ but, as expected, there was no difference between MTII and PF. B relative food intake in grams per gram of BW during the course of the experiment. Both MTII and PF had reduced caloric intake relative to Control group $(P s<0.01)$ and, again, there was no difference between MTII and PF. All values represent the mean \pm SEM of 4 rats per group (MTII) or 5 rats per group (Control and PF).

\section{Fig. 2 Periodic measures of cumulative food intake and delta body mass}

Total food consumed or changes in delta $(\Delta)$ body mass between day 0 and 14, between day 14 and 28, and between day 0 and 28. A Over the first 14 days, MTII and PF animals ate significantly less food than Control $(P<0.01)$ but there was no difference between MTII and PF. B There was no difference across groups in the amount of food consumed between over the last 14 days $\mathbf{C}$ There was no difference across groups in the amount of food consumed throughout the study. D Body mass was significantly reduced from day 0 to 14 in both MTII and PF 
animals $(P<0.01)$ but there was no difference between MTII and PF groups. E There was no difference across groups in delta body mass between day 14 and 28. F Body mass was significantly reduced in MTII animals compared with Control and PF $\left(P_{\mathrm{s}}<0.05\right)$ but there was no difference between PF and Control groups. All values represent the mean \pm SEM of 4 rats per group (MTII) or 5 rats per group (Control and PF). * Significantly different from Control $(P<0.05), * *(P<0.01), * * *(P<0.001) . \&$ Significantly different from MTII $(P<0.05)$.

\section{Fig. 3 Longitudinal changes in body composition}

Changes in fat mass $(\Delta \mathrm{FM})$, changes in lean body mass $(\Delta \mathrm{LBM})$, and lean:fat mass ratio were recorded at day 0,14, and 28. A MTII group lost a significantly higher amount of fat at day 14 $(P s<0.001)$ and day $28(P s<0.05)$ compared with Control and PF rats. B MTII and PF rats lost a significant amount of LBM compared with Control $(P s<0.05)$ by day 14 . However, by day 28 , only MTII rats displayed significantly higher LBM loss than Control $(P<0.05)$ but there was no significant difference between MTII and PF. C At day 0, lean/fat mass ratio was similar across groups. At day 14, PF animals exhibited a significantly lower lean/fat mass ratio than Control and MTII rats $(\mathrm{P}<0.05)$ but no differences were observed at day 28. All values represent the mean \pm SEM of 4 rats per group (MTII) or 5 rats per group (Control and PF). * Significantly different from Control $(P<0.05), * *(P<0.01), * * *(P<0.001)$. \& PF significantly different from MTII $(P<0.05), \& \&(P<0.01)$. 

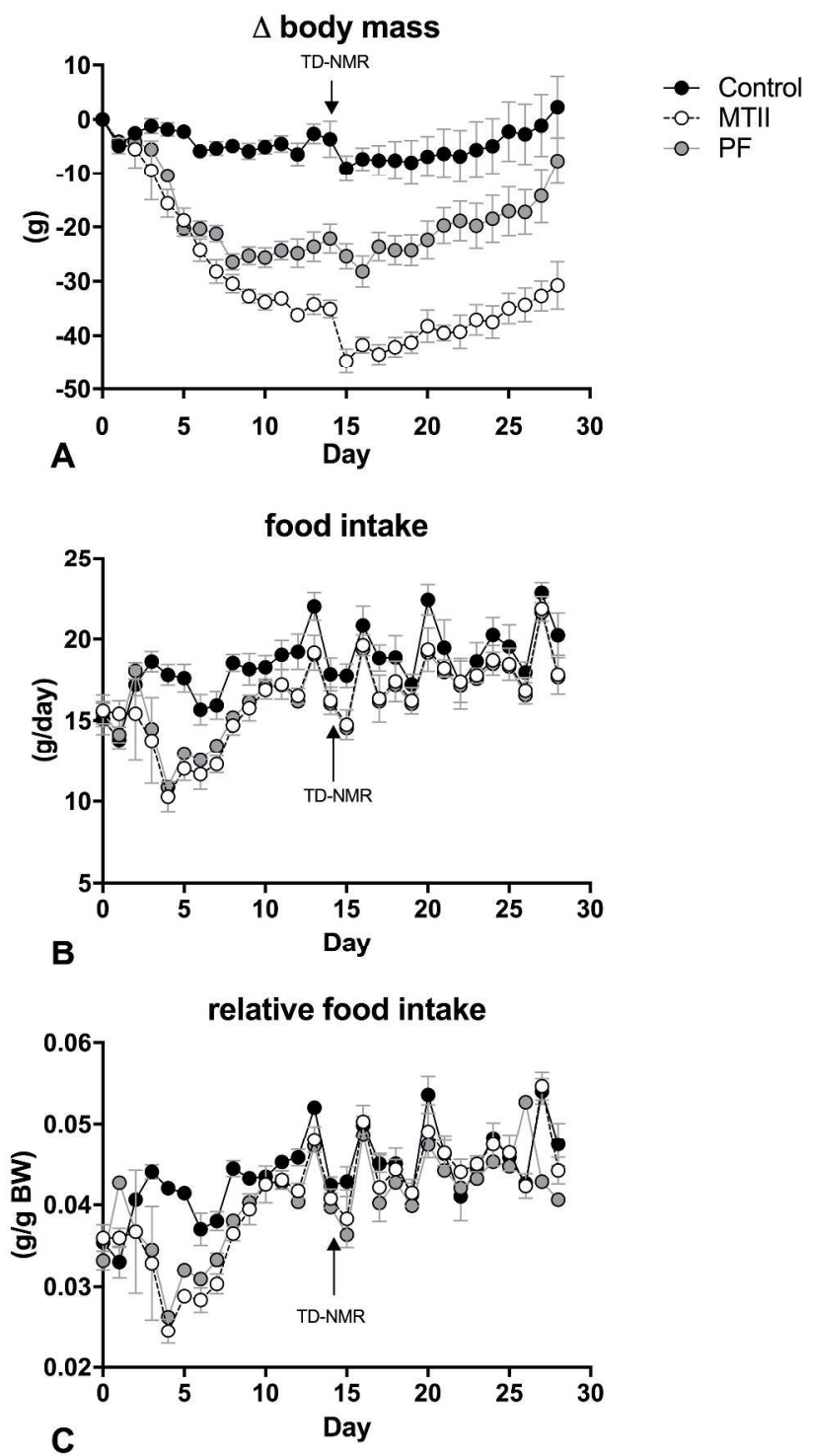

Fig. 1

Fig. 1 Longitudinal changes in body mass and daily food intake $221 \times 387 \mathrm{~mm}(300 \times 300 \mathrm{DPI})$ 

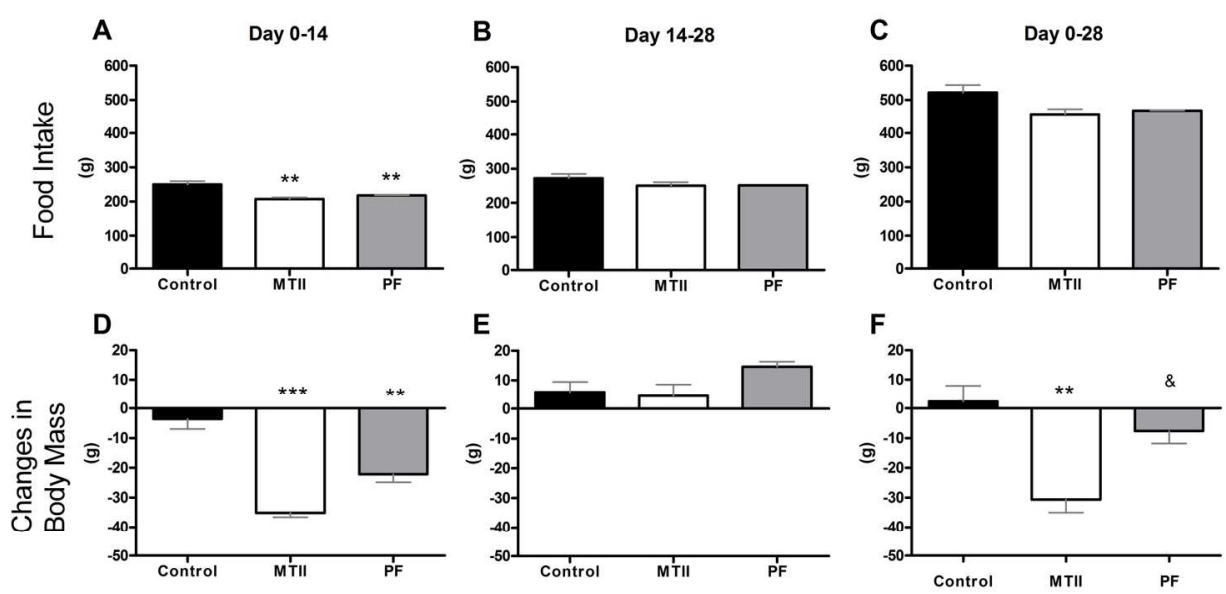

Fig. 2

Periodic measures of cumulative food intake and delta body mass $164 \times 85 \mathrm{~mm}(300 \times 300$ DPI $)$ 


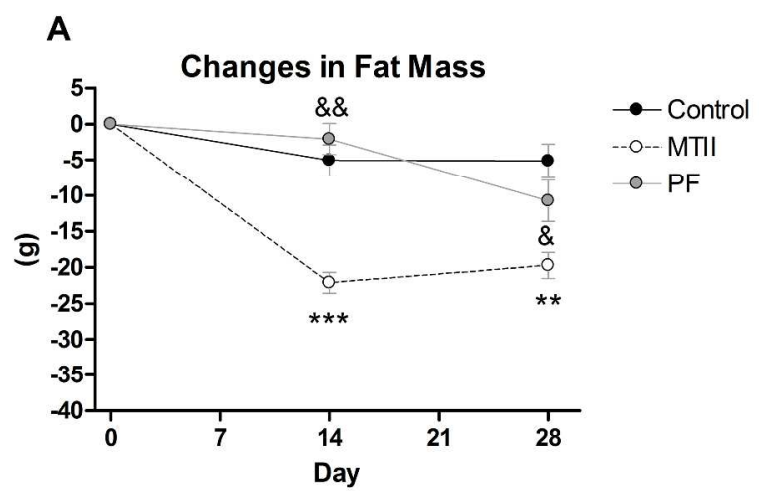

B
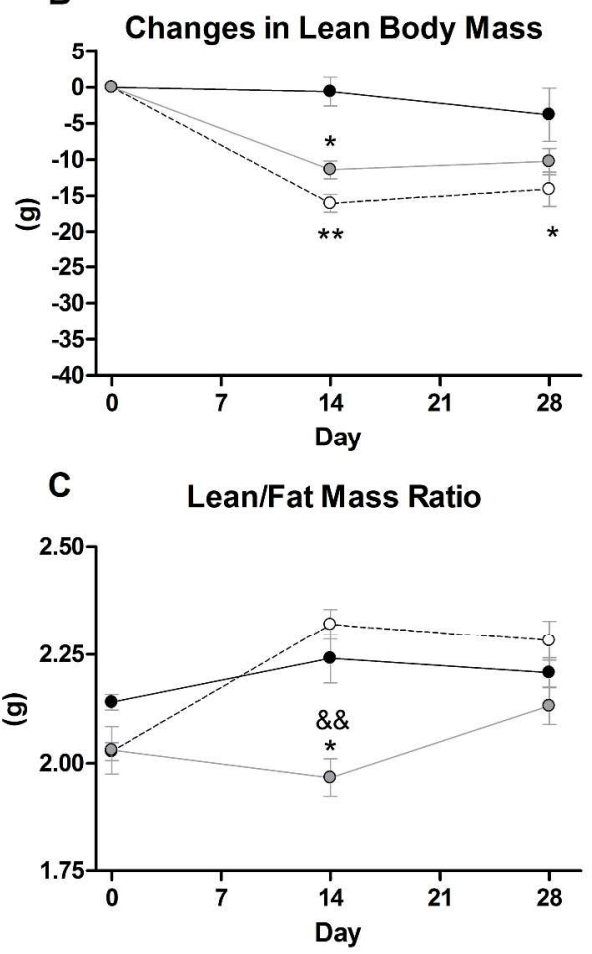

Fig. 3

Longitudinal changes in body composition $301 \times 553 \mathrm{~mm}(300 \times 300$ DPI $)$ 